\title{
The Hazards Posed by Mesoscale Lightning Megaflashes $\mathscr{O}$
}

\author{
Michael Peterson ${ }^{\mathrm{a}}$ AND GeOFFrey STANO ${ }^{\mathrm{b}}$ \\ ${ }^{\text {a }}$ Space and Remote Sensing Group, Los Alamos National Laboratory, Los Alamos, New Mexico \\ ${ }^{\mathrm{b}}$ University of Alabama in Huntsville, Huntsville, Alabama
}

(Manuscript received 14 September 2020, in final form 12 April 2021)

\begin{abstract}
Lightning megaflashes extending over $>100-\mathrm{km}$ distances have been observed by the Geostationary Lightning Mappers (GLMs) on NOAA's R-series Geostationary Operational Environmental Satellites (GOES). The hazards posed by megaflashes are unclear, however, because of limitations in the GLM data. We address these by reprocessing GOES-16 GLM measurements from 1 January 2018 to 15 January 2020 and integrating them with Earth Networks Global Lightning Network (ENGLN) observations. ENGLN verified 194880 GLM megaflashes as natural lightning. Of these, 127479 flashes occurred following the October 2018 GLM software update that standardized GLM timing. Reprocessed GLM/ENGLN lightning maps from these postupdate cases provide a comprehensive view of how individual megaflashes evolve. This megaflash dataset is used to generate statistics that describe their hazards. The average megaflash produces 5-7 cloud-to-ground (CG) strokes that are spread across $40 \%-50 \%$ of the flash extent. As flash extent increases beyond $100 \mathrm{~km}$, megaflashes become concentrated in key hot-spot regions in North and South America while the number of CG and intracloud events per flash and the overall peak current increase. CGs in the larger megaflashes occur over $80 \%$ of the flash extent measured by GLM, and the majority contain regions where the megaflash is the only lightning activity in the preceding hour. These statistics demonstrate that there is no safe location below an electrified cloud that is producing megaflashes, and current lightning safety guidance is not always sufficient to mitigate megaflash hazards.
\end{abstract}

KEYWORDS: Lightning; Thunderstorms; Satellite observations

\section{Introduction}

While lightning occurs most frequently in intense convection, the overall lightning hazard encompasses all surrounding regions where an individual or an operation might be adversely affected by lightning. The lightning hazard differs according to which part of the thunderstorm is being considered. Lightning is common in the convective core (Peterson and Liu 2011) where other hazards such as hail and strong winds exist that motivate individuals to seek shelter. Lightning flash rate trends are symptomatic of updraft characteristics (Deierling and Petersen 2008) and ice fluxes (Deierling et al. 2008), and sudden increases (i.e., "jumps") in lightning activity have been used to predict the onset of severe weather (Williams et al. 1999; Schultz et al. 2009).

The 30-30 rule for lightning safety that was proposed by a "lightning safety group" at the Annual Meeting of the American Meteorological Society (AMS) in 1998 (Holle et al. 1999) works best with this convective-type lightning. By this rule, lightning is considered dangerous if the time difference between the flash of light and the clap of thunder is less than

Supplemental information related to this paper is available at the Journals Online website: https://doi.org/10.1175/EI-D-200016.s1.

Corresponding author: Michael Peterson, mpeterson@lanl.gov

Earth Interactions is published jointly by the American Meteorological Society, the American Geophysical Union, and the Association of American Geographers.
$30 \mathrm{~s}$. This delay is due to the difference between the speed of light and the speed of sound in air and works out to describe a lightning strike within $\sim 10 \mathrm{~km}$ of the observer. Holle et al. (1999) noted the 30-s "flash to bang" part of the rule was insufficient for certain types of lightning. Lopez and Holle (1999) suggested that greater distances should be considered for large, organized convective systems. This creates a problem for the perception of danger, however. They note that lightning is not perceived to be close to the observer when longer flash-to-bang times are used. This can lead the observer to not appreciate the risk until the next strike occurs at their location. Moreover, the perception of low risk is amplified when the apparent flash rate is low-with minutes between visible strokes. Because of these limitations, some organizations do not recommend using the 30-30 rule. For example, the guidance provided by NOAA recommends seeking shelter on any detection of thunder (i.e., "when thunder roars, go indoors"; NOAA 2018). If the lightning is close enough that an observer can hear the audible shockwave it generates, then it is potentially close enough to strike them.

Lightning flashes outside the convective core pose a unique hazard as compared with convective lightning. This is because there exists a natural opposition between flash frequency and flash size (Bruning and MacGorman 2013). While the heterogeneous electric field in the convective core produces high rates of relatively small flashes, homogeneous nonconvective electrified clouds are infrequently discharged by lightning flashes that develop laterally over long horizontal distances. The overall maximum size of the flash is only limited by the extent of the charge reservoir that it can access in the electrified cloud. Large flashes are particularly common in mesoscale convective systems (MCSs), while the largest cases occur 
exclusively in these organized convective systems. MCSs are favorable for large lightning because they produce electrified stratiform regions that can extend over hundreds of kilometers (Marshall and Rust 1993; Stolzenburg et al. 1994; Lang et al. 2004) through charge advection from the convective line (Carey et al. 2005) and in situ generation (Rutledge and MacGorman 1988; Ely et al. 2008; Lang and Rutledge 2008). These long horizontal lightning flashes have been termed "megaflashes" (Lyons et al. 2020) and are defined as a mesoscale lightning flash that is at least $100 \mathrm{~km}$ long.

The factors that describe nonconvective lightning hazards-long horizontal flashes occurring in low flash rate regions of larger organized storm systems - are each, individually, conducive to an underappreciated lightning threat. Their combination is an ideal mix for a "bolt from the blue" if under clear skies or a "bolt from the gray" (Lyons et al. 2020) if under cloudy skies. Perhaps the storm passed long ago with only low stratiform clouds remaining. Then, suddenly, a lightning flash comes from over the horizon and streaks across the sky putting down multiple cloud-to-ground (CG) strokes along its path. Those stratiform clouds overhead were electrified and, even though they were not actively flashing on their own, they still serve as a charge reservoir for lightning initiated elsewhere (Marshall and Rust 1993; Lang et al. 2004; Carey et al. 2005). Further adding to the hazard, this type of lightning often produces positive $\mathrm{CG}(+\mathrm{CG})$ strokes with high peak currents and continuing current (CC) that lead to large charge moment changes. The physical attributes of these strokes are favorable for initiating forest fires (Latham and Williams 2001) and generating exotic upper-atmosphere electrical discharges such as sprites (Franz et al. 1990; Williams 1998; Lyons et al. 2009; Williams et al. 2010).

Scenarios as described above have been documented for individual cases of megaflashes that were mapped from space by NOAA's Geostationary Lightning Mapper (GLM; Goodman et al. 2013; Rudlosky et al. 2019). GLM is the first operational lightning detector that can continuously map individual flash extent over broad (hemispheric scale) geospatial domains. Groundbased radio-frequency (RF) lightning networks including the National Lightning Detection Network (NLDN) resolve the locations of strokes and some cloud pulses, but these sparse detections are not sufficient to resolve megaflash structure. Lyons et al. (2020) showed an impressive megaflash case in which the most distant NLDN events associated with the GLM flash were $500 \mathrm{~km}$ apart-starting on the Oklahoma-Texas border and ending in central Kansas. The strongest $+\mathrm{CG}$ strokes had peak currents exceeding $300 \mathrm{kA}$ and charge moment changes $>3100 \mathrm{C} \mathrm{km}$ (well within the range for sprite production).

Individual case studies are instructive for demonstrating what megaflashes are capable of but documenting the lightning hazard posed by megaflashes requires taking a statistical approach. The geostationary orbit of the GOES satellites allows GLM to record a staggering amount of lightning data. The GOES-16 GLM detects on the order of a million lightning flashes per day. Each year of GOES-16 GLM observations includes around 365 million flashes, which is nearly 10 times as much lightning as the 38 million flashes that NASA's Optical Transient Detector (OTD) and Lightning Imaging Sensor
(LIS) instruments could have observed (i.e., after correcting for instrument detection efficiency) during their combined 25 years in low Earth orbit.

Unfortunately, the operational GLM data do not permit megaflashes to be identified routinely. Strict latency requirements have resulted in hard limits being imposed by the operational GLM ground system software (Goodman et al. 2012) for the maximum complexity and duration of a single lightning flash. When a flash exceeds either 101 "groups" (an approximation for individual optical pulses) or $3 \mathrm{~s}$ in duration, it will be terminated by the ground system software and any additional detections will be assigned to a new flash. This results in megaflashes being artificially split into dozens of smaller flashes.

To identify these megaflash cases, the operational GLM lightning data need to be reprocessed to resolve each complete and distinct lightning flash. We employ a "reclustering" approach (Peterson 2019) that evaluates the clusters in the operational GLM data produced by NOAA, identifies any flashes that contain groups that should be clustered into the same flash, and then merges the split flashes into a single flash cluster. The largest case of natural lightning in the reclustered GLM dataset was a 709-km megaflash that recently has been recognized by the World Meteorological Organization (WMO) as the global lightning extreme for flash extent (Peterson et al. 2020a). Another 16.73-s flash in this dataset was also recognized by the $\mathrm{WMO}$ as the global lightning extreme for flash duration.

In the present study, we integrate ground-based RF lightning measurements with our reclustered GLM dataset to document the lightning hazard posed by megaflashes across the Americas. As with Lyons et al. (2020), the RF measurements provide information on the ground strike locations and peak currents that are not measured by GLM. We use this combined dataset to produce statistics on the number of strokes per megaflash, the peak current and polarity of megaflash strokes, and the fraction of the megaflash horizontal extent where ground strikes occur. These statistics reinforce the unpredictable nature of the megaflash lightning hazard. Ground strikes can occur anywhere within the megaflash extent and frequently have high peak currents that are capable of causing damage, injury, or igniting fires. As suggested by Lopez and Holle (1999), greater care should be taken with organized convective systems-especially when near electrified anvil and stratiform clouds that are capable of producing a megaflash. Lightning in these regions may be infrequent, but it only takes one unexpected lightning flash to spark a tragedy.

\section{Data and method}

\section{a. GLM data}

Megaflashes are identified in the reclustered GOES-16 GLM science data described at length in Peterson (2019) and more recently in Peterson et al. (2020a). This reprocessed dataset extends from 1 January 2018 until 15 January 2020 and includes the whole GOES-16 GLM domain that covers North and South America from $54^{\circ} \mathrm{S}$ to $54^{\circ} \mathrm{N}$.

GLM detects lightning as transient increases in cloud illumination at the 777.4-nm oxygen emission triplet. The GLM 
domain is imaged at 500 frames per second on a $1372 \times 1300$ pixel charge-coupled device (CCD) imaging array. The GLM imaging array features variable-pitch pixels that maintain a relatively consistent horizontal resolution projected to ground ranging from $8 \mathrm{~km}$ at nadir to $14 \mathrm{~km}$ at the limb. The steadystate radiant energy of the background scene at each pixel is subtracted from the instantaneous pixel energy, and then an "event" is registered if this difference exceeds the threshold for detection (Rudlosky et al. 2019).

The GLM data are organized into a hierarchy of cluster features that describe lightning over a range of temporal and spatial scales. Individual events during a single integration frame are the basic unit of GLM detection. Events do not represent complete physical processes, but rather describe locations on the CCD array that light up during lightning phenomena. Events are clustered into "groups" that describe contiguous regions on the CCD array that light up simultaneously. Thus, groups approximate cloud illumination from individual optical pulses generated by lightning. This is only an approximation because the 2-ms duration of GLM integration frames is considerably larger than the duration of individual optical pulses (usually on the order of $100 \mu \mathrm{s}$ ). Thus, the possibility exists that a single GLM group might capture multiple pulses. On the other hand, CC generates sustained optical emission that would last for multiple 2-ms GLM groups.

Groups that are close in space and time are then clustered into higher-level features that describe distinct lightning flashes. The process for constructing flashes is based on the clustering technique employed with LIS and validated over its $17-\mathrm{yr}$ mission on the Tropical Rainfall Measuring Mission (TRMM) satellite (Mach et al. 2007). For LIS, group centroids were evaluated for flash assignment by a three-term weighted Euclidean distance (WED) model applied in geolocated space. The three terms were the zonal difference in position (DX) between groups, the meridional difference in position (DY), and the time difference (DT). The spatial terms were weighted by a threshold of $5.5 \mathrm{~km}$, and the temporal term was weighted by $330 \mathrm{~ms}$. If two groups fell within the sphere defined by WED $=1$, then they were determined to belong to the same flash.

The GLM clustering algorithm described in Goodman et al. (2013) differs from this LIS algorithm in two key ways. First, rather than using the group centroid locations as the basis for clustering, the GLM algorithm considers the positions of all events that constitute the group feature. If any of these events satisfy the WED model with an event in another group, they will be clustered into the same flash. The second key difference is the spatial threshold chosen. GLM uses the same $16.5-\mathrm{km}$ threshold that was employed with the OTD instrument instead of the 5.5-km LIS threshold to accommodate the larger 8-14km GLM pixels. Mach (2020) evaluated the clustering scheme used for GLM and found that variations in algorithm thresholds did not lead to large changes in the resulting flash rates for all but the most active thunderstorms ( $>40$ flashes per minute).

The reclustered GLM data aim to extend the standard operational GLM data while preserving its structure and conventions. This postprocessing evaluates the flash clusters generated by the GLM ground system as described above, identifies cases where flashes are artificially split by the hard limits in flash group count (101) and flash duration (3 s) coded into the ground system software, and then merges the split flash features together into a single distinct and complete flash feature. This processing also adds two feature levels to the GLM hierarchy that are not implemented in the ground system processing. "Area" features that approximate thunderstorm snapshots in the former LIS/OTD data are added that combine flashes in close spatial and temporal proximity into a single feature. "Series" features (Peterson et al. 2017) are also added that describe distinct periods of sustained optical emission from a single flash. Also, the postprocessing adds flash metrics including flash extent (Peterson et al. 2018) and optical multiplicity (Peterson and Rudlosky 2019) and constructs gridded products - such as flash extent density (FED; Lojou and Cummins 2005) and convective probability (Peterson et al. 2020b) — that are packaged alongside the lightning cluster feature data.

In this study, the maximum flash extent, defined as the maximum great circle distance between any two group centroids in a single flash, will be used to identify megaflashes. Any flash that exceeds $100 \mathrm{~km}$ in extent will be designated a megaflash. Because of the meandering nature of long horizontal lightning channels, the actual flash length would likely be greater. However, space-based instruments like GLM are limited in the level of detail that they can resolve with their kilometer-scale pixels, and methods that attempt to quantify the unique flash length (i.e., not counting reillumination) are computationally expensive (Peterson et al. 2018). Identifying megaflashes using a $100-\mathrm{km}$ extent threshold is a computationally inexpensive way to ensure that smaller flashes are not included in the sample, but smaller megaflashes with total lengths $>100 \mathrm{~km}$ and extents $<100 \mathrm{~km}$ will be missed.

\section{b. ENGLN data}

Beyond the issue of flash length versus flash extent, there are two key caveats in using the GLM data to identify megaflashes and the hazard that they pose. First, GLM does not report the locations of ground strikes. GLM is a total lightning detector that cannot reliably differentiate individual intracloud (IC) discharges from CG strokes. Ground networks excel at identifying the locations and times of strokes. Combining GLM and ground network observations mitigates the lack of GLM stroke information and informs the origins of the optical pulses recorded by GLM. Second, the GLM data contains artifacts from solar contamination that can masquerade as megaflash activity (Peterson 2020). These flashes can be additionally screened by looking for a lack of coincidence with ground network observations. Thus, both key caveats are mitigated through data fusion with a ground network.

In this study, Earth Networks Global Lightning Network (ENGLN) data are acquired from Earth Networks and integrated into the GLM clustering hierarchy for the megaflash cases. ENGLN is a distributed heterogeneous global network of long-range ground-based RF lightning sensors. ENGLN integrates observations from two networks: the Earth Networks Total Lightning Network (ENTLN; Zhu et al. 2017) and the WorldWide Lightning Location Network (WWLLN; Jacobson et al. 2006; Lay et al. 2004; Rodger et al. 2006; Hutchins et al. 2012). ENGLN 


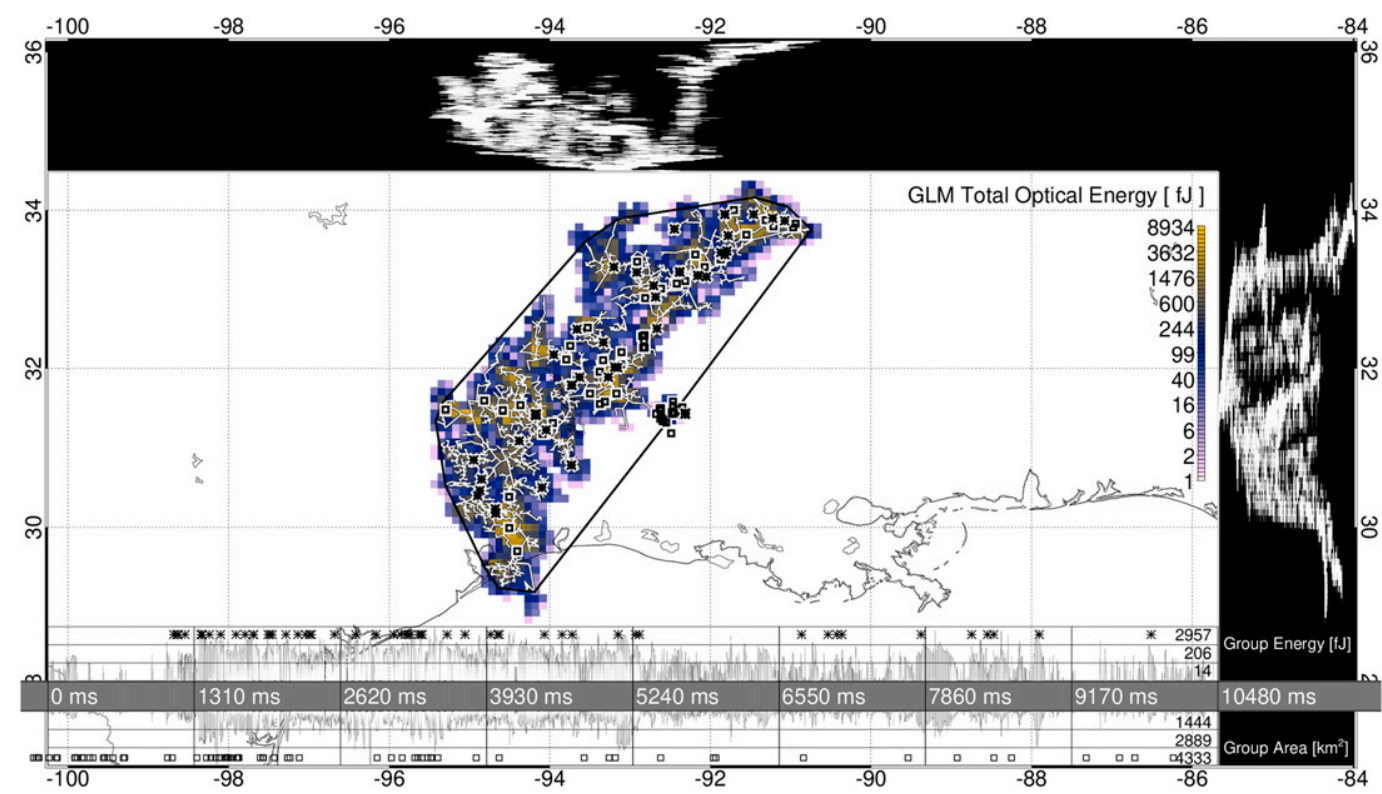

FIG. 1. An example GLM megaflash with coincident ENGLN events added. GLM total optical energy is mapped as a color contour. The group-level structure is overlaid with white line segments on the map, and the latitude and longitude extents of the time-ordered groups are depicted to the right of and above the map. Time series of group energy (above the time axis) and area (below) are shown below the map. ENGLN CG strokes are depicted as asterisks in both the map and time series, and IC events are drawn as box symbols.

data include the position and time of lightning events, their type (CG or IC), and also their peak current and polarity. However, note that distant $+\mathrm{CG}$ strokes can be reported as $-\mathrm{CG}$ if the ground wave becomes attenuated. Thus, $-\mathrm{CGs}$ reported from megaflashes might, in fact, be mis-classified + CGs.

\section{c. Adding ENGLN events to GLM megaflashes}

Our approach for clustering the ENGLN data into the GLM data tree assumes that 1) all ENGLN reports (CG or IC) that are collocated with a GLM group contribute optical energy to that group, and 2) not all ENGLN reports will lead to GLM groups (e.g., if the cloud is too optically thick to allow transmission to space that are bright enough for GLM to detect). Thus, ENGLN reports should be close to the GLM events that comprise groups in space and time, but some leeway should be granted to limit the number of missed reports.

We treat ENGLN events as "groups" (approximating complete lightning pulses) for clustering purposes and assign them to GLM flashes if they occur within $16.5 \mathrm{~km}$ and $500 \mathrm{~ms}$ of any GLM event within one of the groups from that flash. We use the box-distance clustering algorithm from OTD rather than the WED method used by LIS and GLM to reduce computational expense. While this clustering approach is applied to all ENGLN events that share coincidence with GLM megaflashes, it is important to note that the rates of matched events are not uniform in space and time. The GLM operating software was updated multiple times during our 2-yr period in the reprocessed GLM record (2018-20), some of which improved the geolocation and timing accuracy in the later portion of the data record. These changes have minimal impact on whether a GLM megaflash contained an ENGLN event but will affect the number and locations of matched ENGLN events in a given GLM flash. Thus, we focus our assessment of matched GLM megaflash characteristics on the 31 October 2018-15 January 2020 period with the best timing information.

The other major factor impacting clustering uniformity is the fact that ENGLN does not have a uniform sensor density. Dense observations permit more events (especially weaker events) to be resolved. The sensor density is greatest in the United States, and the ENTLN domain in the surrounding regions contains drastically more events per square kilometer than the remainder of the GOES-16 GLM field of view (FOV). As with GLM timing, this is not expected to impact whether a GLM megaflash will have ENGLN coincidence, but it will influence the number of coincident ENGLN events (especially IC events) per flash and their relative extent in comparison with the GLM flash extent.

Figure 1 shows an example GLM megaflash with ENLGN events added. This particular megaflash over Louisiana was identified in Peterson (2019) as having the greatest unique footprint area reported by GLM, a 634-km overall extent, and a duration of nearly $10.5 \mathrm{~s}$. The groups in this flash (connected by line segments in the central panel) developed westward from the flash start position at the rear of the convective line and then spread in many directions throughout the stratiform region of the MCS. The latitude extent of each time-ordered group in the flash is shown to the right of the map while the longitude extent of each group is shown above the map. A time series of group area (above the time axis) and group energy (below the time axis) is shown along the bottom of the figure. ENGLN CGs are 


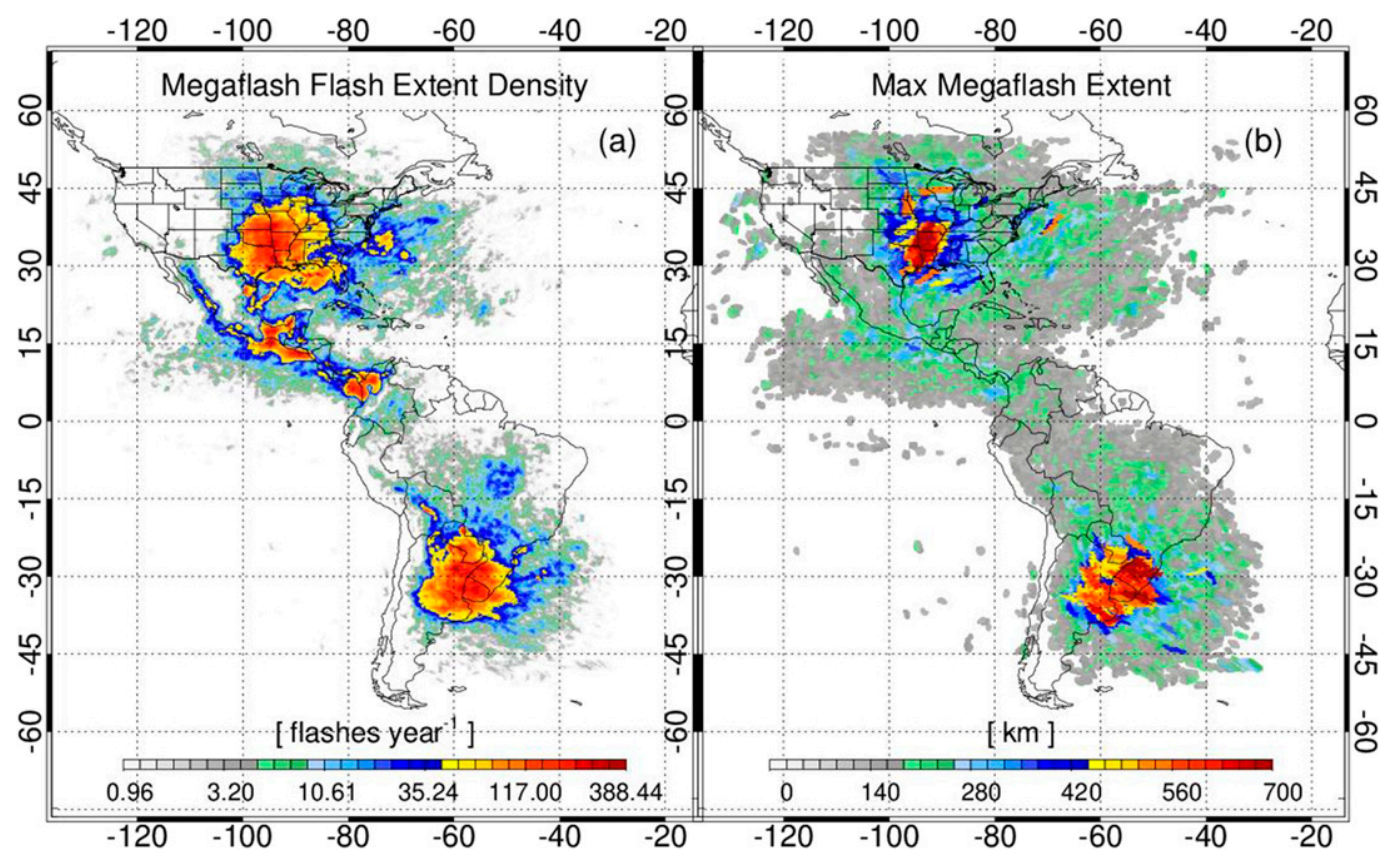

FIG. 2. Hemispheric distributions of GLM megaflash activity depicted as a (a) FED and (b) peak megaflash extent in the 1 Jan 2018-15 Jan 2020 record.

added as asterisk symbols in both the map and the top time series, and ENGLN ICs are depicted as box symbols. In total, 126 ENGLN events were reported during this flash including $36-\mathrm{CG}$ strokes and $17+\mathrm{CG}$ strokes. The first of these strokes occurred $1.126 \mathrm{~s}$ into the flash while the last occurred $0.601 \mathrm{~s}$ before the end of the GLM flash. The strokes were not clustered in a single location, but rather scattered throughout the 114000 $\mathrm{km}^{2}$ footprint of the GLM flash. The strongest - CG stroke from this flash had a peak current of $-118 \mathrm{kA}$, whereas the strongest $+\mathrm{CG}$ stroke had a peak current of $+133 \mathrm{kA}$.

This information about the strokes that occurred during this flash was not available in the previous analysis in Peterson (2019) because it only considered GLM and did not add ground network observations. On the other hand, the ground networks do not map lightning flashes with a sufficient level of detail to identify flash structure-information that is readily available with GLM. Data fusion between these optical and RF measurements from the same flash enable comprehensive assessments of individual megaflashes that are not possible with either phenomenology in isolation.

Our merged GLM-ENGLN data contain 194880 GLM megaflashes like the example in Fig. 1 that were observed between 1 January 2018 and 15 January 2020 across the GOES-16 GLM full-disk domain. These megaflash data are hosted at Peterson (2020b). These flashes are associated with a total of 4.5 million ENGLN events. 1 million of these events (22\%) were from CGs while the remaining 3.5 million events (88\%) were ICs. We will focus, however, on the period with improved GLM timing accuracy (31 December 2018 onward), reducing the size of the megaflash sample considered to 127479 flashes $(65 \%$ of all GLM megaflashes) across the GOES-16 GLM full disk.

\section{Results}

The following sections assess the megaflash lightning hazard. Section 3 a maps the locations and peak extents of ENGLNmatched GLM megaflashes, and then summarizes their overall attributes that define the lightning hazard. Section 3 b elaborates on the statistics of ENGLN matches by examining their frequencies and peak currents as a function of GLM megaflash extent. Finally, section $3 c$ addresses the questions of whether megaflashes pose a risk of CG strikes over their full spatial extent as mapped by GLM, and whether megaflashes are sufficiently isolated in time that the public might have resumed outdoor activities when these flashes occur if following the 30-30 rule.

\section{a. Overall statistics on GLM megaflash location and composition}

Megaflashes may be relatively uncommon in the GLM record in comparison with convective lightning, but there are certain regions in the Americas that produce, on average, oneor-more megaflashes per day. Figure 2a shows the locations of these "hot spot" regions: the Great Plains, Gulf Coast, and Eastern Seaboard of the United States; coastal Central America from Mexico to Colombia; and portions of southern Brazil, Uruguay, Paraguay, Bolivia, and Argentina in South America. Note that Fig. 2 is the only analysis in this study that uses all 194880 ENGLN-matched GLM megaflashes.

While the term "megaflash" is applied to each case of $100+-\mathrm{km}$ lightning, some flashes far exceed this threshold and extend for multiple hundreds of kilometers. These longer megaflashes exhibit notably different behavior than their 100-km counterparts. The first example of this is in Fig. $2 b$, which shows the peak 
TABLE 1. General statistics describing the average number of ENGLN events in GLM megaflashes and their lateral separations.

\begin{tabular}{|c|c|c|c|c|c|c|c|c|c|}
\hline & \multirow{3}{*}{$\begin{array}{c}\text { GLM } \\
\text { flash } \\
\text { count }\end{array}$} & \multirow{2}{*}{\multicolumn{4}{|c|}{$\begin{array}{l}\text { Average ENGLN events per } \\
\text { GLM flash }\end{array}$}} & \multicolumn{2}{|c|}{$\begin{array}{l}\text { Average CG max } \\
\text { separation }\end{array}$} & \multicolumn{2}{|c|}{$\begin{array}{l}\text { Average IC max } \\
\text { separation }\end{array}$} \\
\hline & & & & & & \multirow{2}{*}{$\begin{array}{l}\text { Distance } \\
(\mathrm{km})\end{array}$} & \multirow{2}{*}{$\begin{array}{c}\text { Percent of } \\
\text { flash extent }\end{array}$} & \multirow{2}{*}{$\begin{array}{l}\text { Distance } \\
(\mathrm{km})\end{array}$} & \multirow{2}{*}{$\begin{array}{l}\text { Percent of } \\
\text { flash extent }\end{array}$} \\
\hline & & All & $+\mathrm{CGs}$ & $-\mathrm{CGs}$ & ICs & & & & \\
\hline \multicolumn{10}{|c|}{ GOES-16 GLM field of view } \\
\hline Full disk & 127479 & 23.2 & 1.0 & 4.5 & 17.7 & 51.8 & 37.1 & 75.6 & 56.8 \\
\hline $\begin{array}{l}\text { ENGLN-only } \\
\text { domain }\end{array}$ & 80890 & 13.8 & 0.6 & 3.4 & 9.8 & 41.5 & 29.6 & 60.5 & 45.4 \\
\hline \multicolumn{10}{|c|}{ Subtropical large megaflash $(300+\mathrm{km})$ hot-spot regions } \\
\hline North America & 46576 & 39.6 & 1.6 & 6.5 & 31.5 & 69.8 & 50.2 & 101.8 & 76.6 \\
\hline South America & 50402 & 18.3 & 0.9 & 4.2 & 13.2 & 54.1 & 37.2 & 76.8 & 55.9 \\
\hline
\end{tabular}

megaflash extent across the Americas. While 100-km megaflashes can occur anywhere, the largest flashes observed at most locations across the GLM FOV are $100-200 \mathrm{~km}$ across. The largest megaflashes that have been observed by GLM thus far are 500-700 km in extent (Lyons et al. 2020; Peterson et al. 2020a), and these have only been detected in the Great Plains in North America and the La Plata basin in South America. Megaflashes and their associated hazards might be common in certain coastal and oceanic regions-for example, along the Central American coast-but only these continental basins have been shown to produce MCS thunderstorms capable of generating megaflashes that cover the equivalent land areas of entire states at a time. These large megaflashes have the potential to be particularly dangerous because of their exceptional distance from the convective core of the parent thunderstorm. Locations far removed from the lightning maxima in the storm core may be interpreted as having a low risk for lightning impacting outdoor activities. However, as long as these outlying clouds remain electrified, they can provide a conduit for megaflashes to strike "out of the gray."

The lightning hazard posed by megaflashes, in general, is quantified in Table 1. To improve the likelihood of matching GLM and ENGLN events, only the 31 October 2018-15 January 2020 data (described in section 2c) are used from this point forward. The average megaflash across the GOES-16 GLM fulldisk domain contains 23.2 ENGLN events that include 4.5-CGs, $1+\mathrm{CG}$, and 17.7 IC pulses. When CG strokes are detected, their average maximum separation is $51.8 \mathrm{~km}$, or $37.1 \%$ of the overall GLM flash extent. When IC strokes are detected, their average maximum separation is $75.6 \mathrm{~km}$, or $56.8 \%$ of the GLM flash extent.

Despite using only the most recent GLM data to make these assessments, these numbers still underrepresent the megaflash hazard due to the inclusion of sparse ENGLN observations far from the dense portion of the network. The ENGLN-only region outside the United States has fewer CGs and ICs per GLM flash that are spread over notably smaller fractions of the GLM extent. However, it is possible that this is due to the physical differences between land-based and oceanic or tropical and subtropical megaflashes rather than just ENGLN detection efficiency. Thus, Table 1 specifically compares the continental hot-spot regions in North and South America that both contain large and complex megaflash cases. Megaflashes in the North America hot spot (within the ENTLN domain) contain 1.6 times the number of CGs and 2.3 times the number of IC pulses than their South American counterparts. The CGs in these North American hot-spot flashes extend over half the GLM flash extent, while the ICs extend over $77 \%$ of the GLM flash extent.

The statistics in Table 1 show that megaflashes are not only able to generate multiple ground strikes along their path, but that these CGs are also separated by a significant portion of the flash extent measured by GLM. This demonstrates that the lightning hazard is not limited to the regions surrounding the convective core of the thunderstorm. However, GLM flashes within the ENTLN domain are resolved in greater detail by ENGLN than the flashes outside this domain. Thus, the lightning hazard posed by megaflashes outside the ENTLN domain may be underrepresented in some cases. For this reason, the analyses of how the lightning hazard changes with megaflash extent that will be presented in sections $3 b$ and $3 c$ will only use the data from North America. This includes 41616 megaflashes of the 127479 total cases from 31 October 2018 or later. The same analyses for the full disk are still performed, and these are included as online supplemental material for reference. However, these full-disk analyses are not discussed in the following sections.

\section{b. Megaflash lightning hazards posed by ENGLN event count and peak current}

It was shown in section $3 \mathrm{a}$ that the average megaflash produces multiple CG strokes over its $100+-\mathrm{km}$ extent. However, do longer flashes generate more CGs? With access to a larger charge reservoir, do these longer megaflashes generate greater peak currents that can be particularly hazardous? To answer these questions, we produce twodimensional histograms that catalog megaflashes according to their GLM extent and either their ENGLN event count (Fig. 3) or their ENGLN peak current (Fig. 4). The number of GLM megaflashes in each bin is depicted as a color contour plot. Cumulative distribution functions (CDFs) are also computed for flashes with similar sizes, and line plots are overlaid showing the median (thick solid), 25th- and 75 th-percentile (thin solid), 10th- and 90th-percentile (dashed), and 5th- and 95th-percentile (dotted) values.

The two-dimensional histograms in Fig. 3 show that the ubiquitous $100-\mathrm{km}$ megaflashes in the sample can contain a wide range of ENGLN event counts (from 1 to $>100$ ). The 

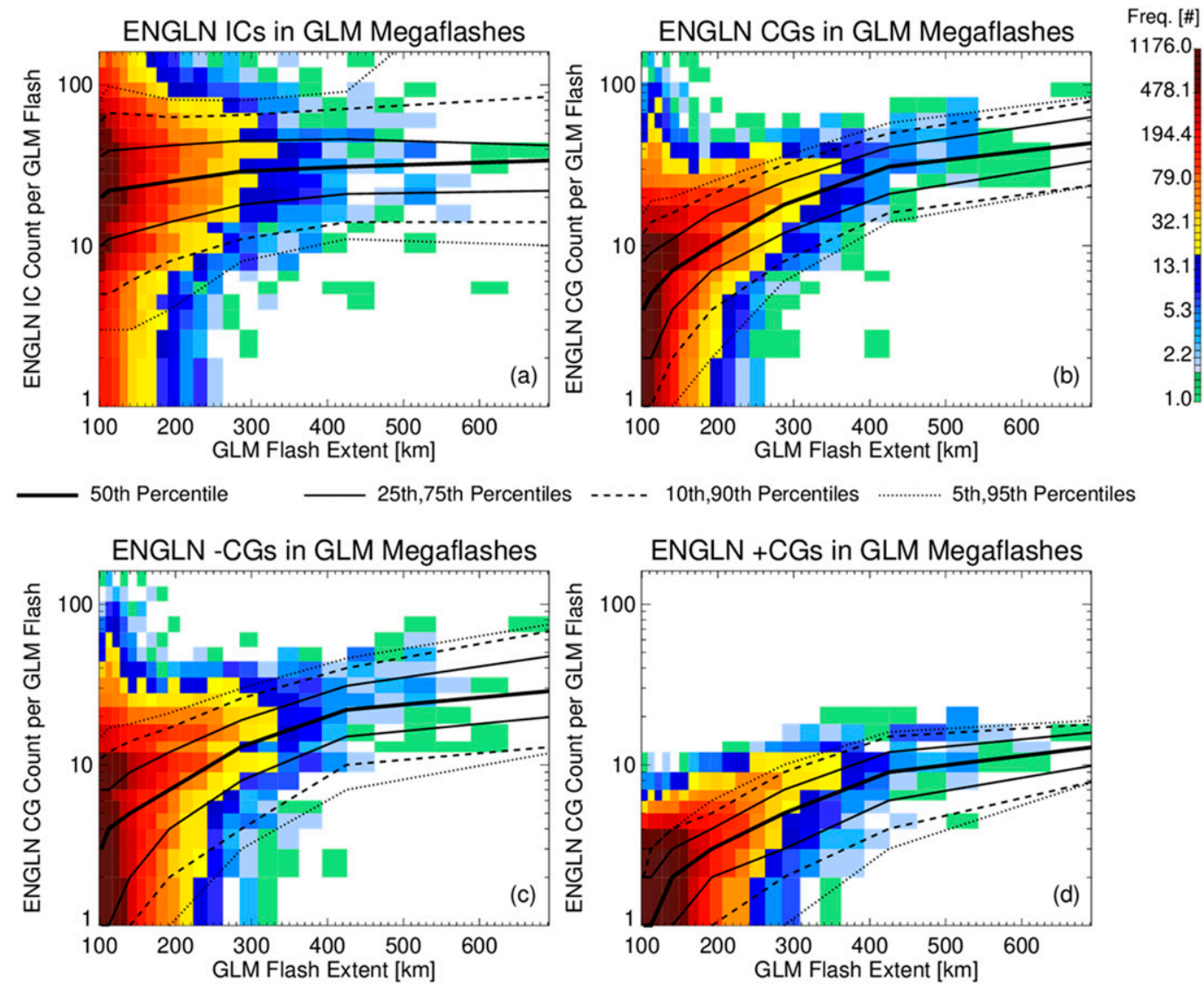

-... 10th,90th Percentiles .............. 5th,95th Percentiles

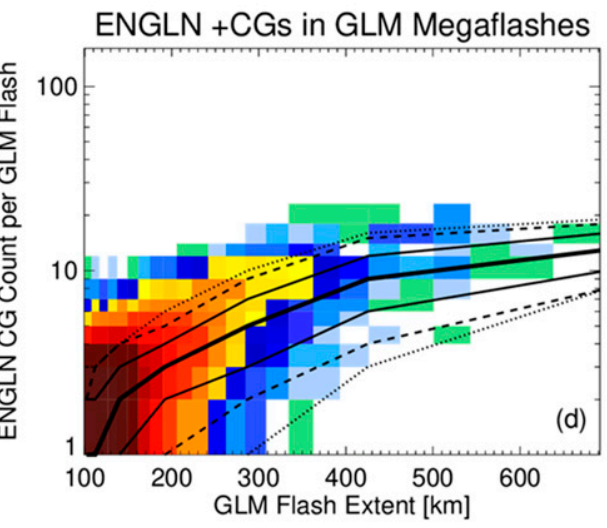

FIG. 3. Two-dimensional histograms (color contours) of GLM flash extent and ENGLN (a) IC count, (b) CG count, (c) - CG count, and (d) + CG count per megaflash. CDFs are produced for GLM megaflashes of similar sizes, and the median (thick solid lines), 25th and 75th percentiles (thin solid lines), 10th and 90th percentiles (dashed lines), and 5th and 95th percentiles (dotted lines) are overlaid.

median number of ENGLN events are 19 ICs (Fig. 3a) and 4 CGs (Fig. 3b, 3 of which are -CGs (Fig. 3c). More than half of the $100-\mathrm{km}$ megaflashes do not produce a +CG (Fig. 3d). As we move up to larger GLM megaflashes, however, the percentile curves shift toward increased numbers of ENGLN events per megaflash. The largest GLM megaflashes have median IC and CG counts of 34 and 45, respectively, while $95 \%$ have at least $11-\mathrm{CGs}$ and $8+$ CGs.
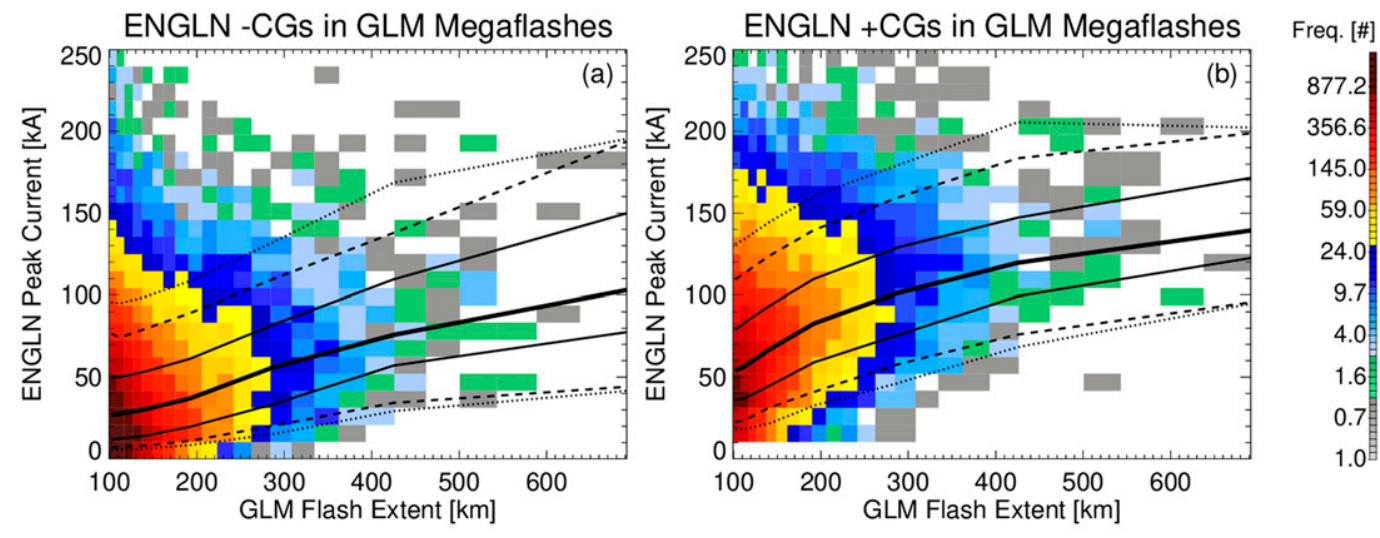

50th Percentile

_ 25th,75th Percentiles

.... 10th,90th Percentiles

.............. 5th,95th Percentiles

FIG. 4. Two-dimensional histograms in the style of Fig. 3, but between GLM flash extent and ENGLN (a) -CG peak current and (b) $+\mathrm{CG}$ peak current. 

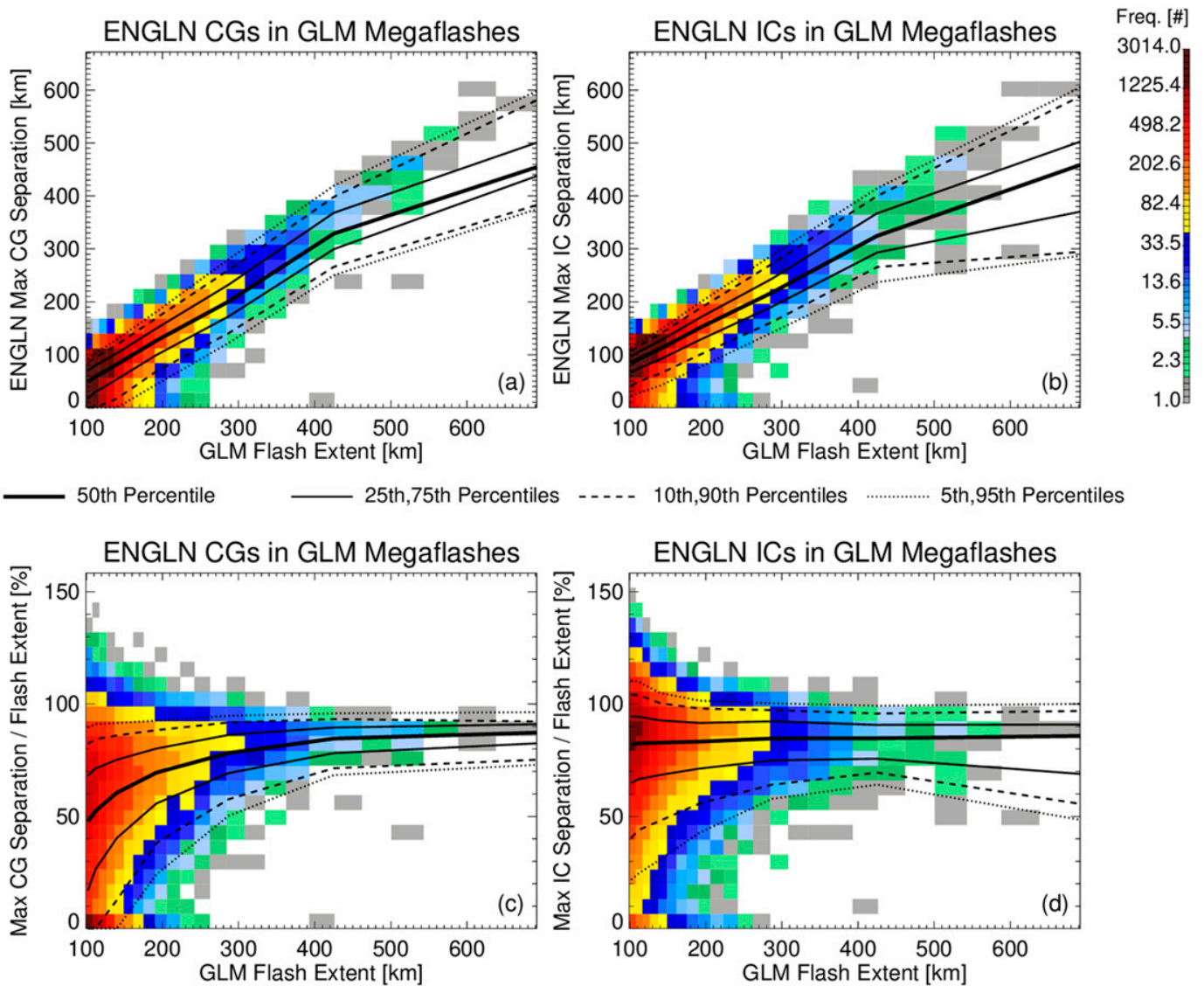

FIG. 5. Two-dimensional histograms in the style of Fig. 3, but between GLM flash extent and ENGLN (a) maximum CG separation distance, (b) maximum IC separation distance, (c) the maximum CG separation distance fraction of the GLM extent, and (d) maximum IC separation distance fraction of the GLM extent.

As the statistics for megaflashes with intermediate extents fall between these two extremes, the risk of multiple megaflash ground strikes only increases with flash extent. At $100 \mathrm{~km}$, there is still a sizable number of megaflash cases with a single CG (Fig. 3b). However, $95 \%$ of megaflashes that are $>140 \mathrm{~km}$ in extent contain multiple CGs and $95 \%$ of megaflashes $>290 \mathrm{~km}$ contain multiple + CGs. Figure 4 shows distributions of ENGLN CG peak current in GLM megaflashes. For all flash extents, megaflash $+\mathrm{CG}$ peak currents are greater than $-\mathrm{CG}$ peak currents. For $100-\mathrm{km}$ megaflashes, $90 \%$ of - CG peak currents and $70 \%$ of + CG peak currents are $<75$ $\mathrm{kA}$. However, by $430 \mathrm{~km}$, over half of $-\mathrm{CG}$ peak currents and $90 \%$ of + CG peak currents exceed $75 \mathrm{kA}$. This is an exceptional peak current threshold, especially for land-based lightning (i.e., Said et al. 2013). Furthermore, $95 \%$ of the largest flashes have + CGs in excess of $93 \mathrm{kA}$. Large flash extents lead to both an increased number of CGs as well as CGs with high peak currents (especially $+\mathrm{CGs}$ ).

\section{c. Megaflash lightning hazards posed by ENGLN event extents and GLM flash rates}

A megaflash generating multiple CGs does not guarantee that strikes can happen throughout its enormous extent.
Moreover, the rarity of megaflashes does not, necessarily, mean that they occur in isolation from other types of lightning. Over what fraction of the megaflash extent does the risk of a ground strike exist? How often do megaflashes exist in regions where someone observing the $30-30$ rule would be caught off guard?

Figure 5 shows two-dimensional histograms that compare GLM flash extent with the maximum separation of ENGLN CG strokes (Figs. 5a,c) and ENGLN IC events (Figs. 5b,d) following the conventions of Figs. 3 and 4. These comparisons are made in terms of absolute great circle distance (Figs. 5a,b) and as a fraction of the megaflash extent resolved by GLM (Figs. 5c,d). The ENGLN maximum event separations increase nearly linearly with GLM events for both CGs (Fig. 5a) and ICs (Fig. 5b). While the detected ENGLN events can be concentrated in a small portion of the megaflash (especially in the smaller 100-km megaflashes), ENGLN CGs and ICs usually exist throughout the megaflash extent measured by GLM.

Table 1 showed that the peak separation of ENGLN CGs is only $50 \%$ of the GLM extent, overall. However, half of $330+-$ $\mathrm{km}$ megaflashes have ENGLN CGs spread across $80 \%$ of their GLM extent and nearly $95 \%$ of the largest GLM megaflashes have ENGLN CGs covering three-quarters of their extent. 

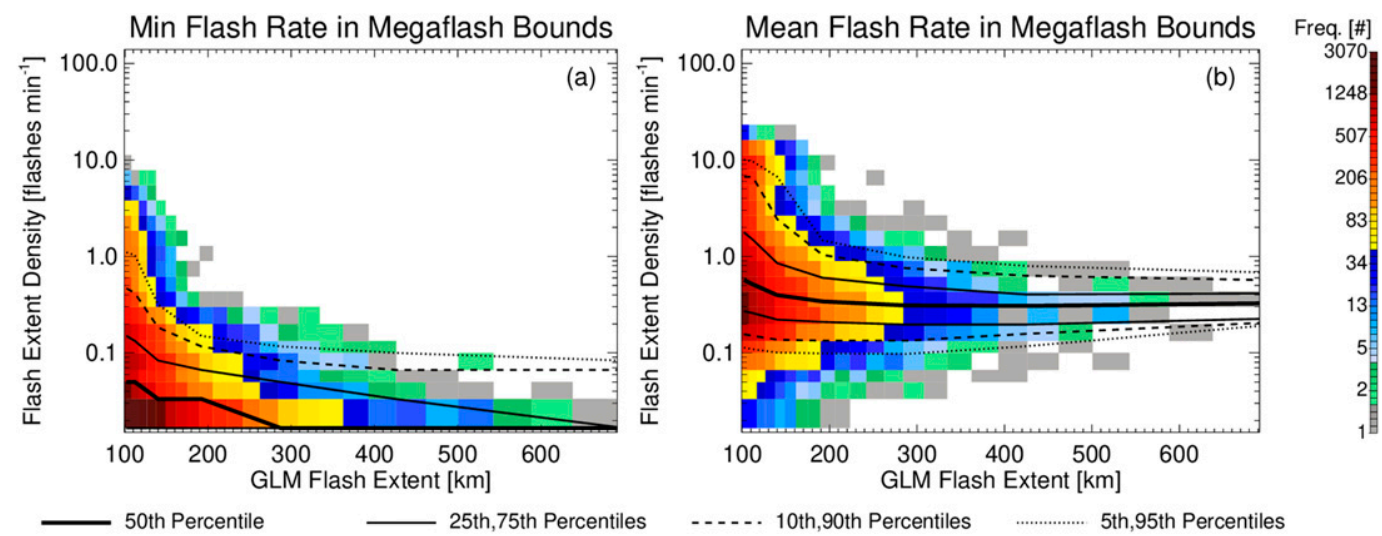

FIG. 6. Two-dimensional histograms in the style of Fig. 3, but between GLM flash extent and GLM (a) minimum flash rate and (b) mean flash rate within the boundaries of each megaflash. Flash rate is quantified as an FED.

Median IC separations (Fig. 5d) are near this $80 \%$ fraction of the GLM flash extent over the full range of flash sizes. If a GLM flash is observed to be $700 \mathrm{~km}$ in extent, one can reasonably expect ENGLN CG and IC sources to occur over a 400-600-km distance. Therefore, it should not be assumed that a safe region exists below an electrified cloud that is producing megaflashes. The first portion of the 30-30 rule (which results in only a $10-\mathrm{km}$ standoff distance) is not appropriate for megaflash cases.

But what about the second portion of the 30-30 rule? Is $30 \mathrm{~min}$ from the previous lightning flash a sufficient period of time to wait before resuming outdoor activities? To answer this question, we use GLM FED to quantify the flash rates at all locations within the megaflash footprint. FED is a gridded product that increments a given gridpoint once for every flash that extends into that gridpoint. If even one of the events that comprises a flash occurs over a particular location, then that flash is counted in the local flash rate. Otherwise, the flash is not counted. Also note that because GLM is a total lightning sensor, the FED grids describe both CG and IC flashes. These FED analyses represent a worst-case scenario for testing the 30-min cessation time because it assumes that all ICs will be audible.

Figure 6 shows two-dimensional histograms for the minimum flash rate (Fig. 6a) and the mean flash rate (Fig. 6b) within the boundaries of each GLM megaflash. The minimum value in each plot is one flash per hour (the maximum time period considered in this analysis). A sizable portion of the $100-\mathrm{km}$ megaflashes occur in relatively active thunderstorm regions with minimum (Fig. 6a) and average (Fig. 6b) FED values reaching 10 flashes per minute. More than $95 \%$ of all flashes of each size occur in regions where the average FED flash rate exceeds 1 flash in $10 \mathrm{~min}$. In these cases, the second half of the 30-30 rule would be appropriate. However, Fig. 6a also shows that a sizable fraction of megaflashes extend into regions where they are the only lightning during a 20-30-min period. In fact, half of the $100-\mathrm{km}$ megaflashes that occur in relatively active storm regions also span inactive regions where the 30-min part of the rule is tested. Meanwhile, the majority of larger megaflashes that extend over multiple hundreds of kilometers violate the 30-min part of the 30-30 rule somewhere within their extent. We thus conclude that neither portion of the 30-30 rule is entirely sufficient to ensure safety from megaflash cases.

Recent analyses of a new operational GLM "stoplight" lightning safety product by Stano et al. (2019) came to the same conclusion. The GLM stoplight product was developed in response to requests from emergency managers to have a realtime lightning product that quickly showed the spatial extent of lightning and how long ago the lightning occurred. Specifically, the stoplight product breaks the visualization into three bins of $0-10,11-20$, and $21-30 \mathrm{~min}$. This binning was purposely selected to match with the operational rule of thumb for waiting $30 \mathrm{~min}$ after the last observation of lightning (either visually or audibly). In the course of the product development, a simple grid cell analysis was conducted for the $79 \mathrm{~h}$ of available data. The available interflash times were analyzed in Stano et al. (2019) to identify how often the 30-min time period was violated (i.e., a flash after 30-min in the same location). In total, 7.4 million "flash pairs" were analyzed and in $1.4 \%$ the time between two flashes exceeded $30 \mathrm{~min}$. Another $0.4 \%$ exceeded $40 \mathrm{~min}$. This basic analysis showed that, in a bulk sense, the $30-$ min wait time is valid, but the risk is nonzero.

\section{Conclusions and discussion}

In this study, we quantify the lightning hazard that is posed by megaflashes that propagate horizontally over distances of at least $100 \mathrm{~km}$. These flashes are distinct from normal convective lightning that generally extend only a few kilometers horizontally. The 30-30 rule for lightning safety recommends taking shelter if lightning is followed by thunder within $30 \mathrm{~s}$, and to remain indoors for $30 \mathrm{~min}$ after the last lightning flash has occurred. However, it is important to remember that these times are based on convective lightning flashes. The 30-s flash-to-bang interval equates to lightning within $\sim 10 \mathrm{~km}$ from the observer-only $10 \%$ of the minimum distance covered by megaflash events.

By analyzing the extent of megaflash cases from GLM with the locations of ENGLN strokes, we are able to demonstrate that megaflashes typically strike multiple locations along their 
$100+-k m$ paths. Larger flashes also have CG activity over more of their overall extents. While $100-\mathrm{km}$ flashes may only produce CGs over a 50-km distance, the top megaflashes typically produce CGs over $\sim 80 \%$ of the overall flash extent measured by GLM, and these CGs also have higher peak currents. Since CG strikes are spread throughout a significant portion of the megaflash footprint, it should not be assumed that there is a safe area below an electrified cloud that is producing megaflashes-regardless of the flash-to-bang times that are measured.

The megaflashes observed by GLM occur primarily outside the convective core of the parent thunderstorm. Low flash rates in these regions and large distances from intense convection (reaching $100 \mathrm{~km}$ or more) can cause the lightning hazard to be underappreciated. Most megaflashes contain regions where the megaflash is the only lightning activity noted by GLM in the previous hour. The second half of the 30-30 rule may be adequate for megaflashes over much of their extent (especially close to the convective core), but not all locations impacted by megaflash activity.

These results lead us to conclude that additional caution must be taken with regard to the large and organized convective systems that are known to produce megaflashes-including below electrified anvil and stratiform clouds. There is no true safe distance when dealing with megaflashes that span hundreds of kilometers. If a thunderstorm produces one megaflash, it can probably generate another that might impact different locations where lightning was previously not observed. An operational meteorologist responsible for decision support services, for example, could recommend the cessation of outdoor activities over a larger area than the 30-30 rule suggests behind a thunderstorm that has demonstrated that it is capable of generating megaflashes, and that outdoor activities remain paused until the electrified stratiform cloud has moved out of the area completely or the meteorological conditions are no longer favorable for continued megaflash activity.

In light of the new megaflash measurements enabled by GLM, the time has come for the community to revisit lightning safety guidance by convening a new lightning safety group akin to the one described in Holle et al. (1999). This community review needs to evaluate common guidance standards against emerging lightning research, new lightning detection capabilities, and also updated risk analyses that have become available since 1998.

Acknowledgments. This work was supported by the U.S. Department of Energy through the Los Alamos National Laboratory (LANL) Laboratory Directed Research and Development (LDRD) program under project 20200529ECR. Los Alamos National Laboratory is operated by Triad National Security, LLC, for the National Nuclear Security Administration of the U.S. Department of Energy (Contract 89233218CNA000001). The GLM LCFA and ABI datasets may be obtained from NOAA via their CLASS service (https://www.class.noaa.gov/) (GLM: https://www.avl.class.noaa.gov/saa/products/search?datatype_family= GRGLMPROD; ABI: https://www.avl.class.noaa.gov/saa/products/ search?datatype_family=GRABIPRD). GLM megaflash data are collected in the database in Peterson (2020b). The ENGLN data used in this study were provided by Earth Networks, Inc. (https://www.earthnetworks.com/) and may be ordered from them.

\section{REFERENCES}

Bruning, E. C., and D. R. MacGorman, 2013: Theory and observations of controls on lightning flash size spectra. J. Atmos. Sci., 70, 4012-4029, https://doi.org/10.1175/JAS-D-12-0289.1.

Carey, L. D., M. J. Murphy, T. L. McCormick, and N. W. Demetriades, 2005: Lightning location relative to storm structure in a leading-line, trailing-stratiform mesoscale convective system. J. Geophys. Res., 110, D03105, https://doi.org/ 10.1029/2003JD004371.

Deierling, W., and W. A. Petersen, 2008: Total lightning activity as an indicator of updraft characteristics. J. Geophys. Res., 113, D16210, https://doi.org/10.1029/2007JD009598.

- — J. Latham, S. Ellis, and H. J. Christian, 2008: The relationship between lightning activity and ice fluxes in thunderstorms. J. Geophys. Res., 113, D15210, https://doi.org/ 10.1029/2007JD009700.

Ely, B. L., R. E. Orville, D. C. Lawrence, and C. L. Hodapp, 2008: Evolution of the total lightning structure in a leading-line, trailing-stratiform mesoscale convective system over Houston, Texas. J. Geophys. Res., 113, D08114, https://doi.org/10.1029/ 2007JD008445.

Franz, R. C., R. J. Nemzek, and J. R. Winckler, 1990: Television image of a large upward electrical discharge above a thunderstorm system. Science, 249, 48-51, https://doi.org/10.1126/ science.249.4964.48.

Goodman, S. J., D. Mach, W. J. Koshak, and R. J. Blakeslee, 2012: GLM Lightning Cluster-Filter Algorithm. NOAA NESDIS Center for Satellite Applications and Research Algorithm Theoretical Basis Doc., 73 pp., https://www.star.nesdis.noaa.gov/ goesr/documents/ATBDs/Baseline/ATBD_GOES-R_GLM_ v3.0_Jul2012.pdf.

_, and Coauthors, 2013: The GOES-R Geostationary Lightning Mapper (GLM). Atmos. Res., 125-126, 34-49, https://doi.org/ 10.1016/j.atmosres.2013.01.006.

Holle, R. L., R. E. López, and C. Zimmermann, 1999: Updated recommendations for lightning safety-1998. Bull. Amer. Meteor. Soc., 80, 2035-2042, https://doi.org/10.1175/15200477(1999)080<2035:URFLS>2.0.CO;2.

Hutchins, M. L., R. H. Holzworth, J. B. Brundell, and C. J. Rodger, 2012: Relative detection efficiency of the World Wide Lightning Location Network. Radio Sci., 47, RS6005, https://doi.org/ 10.1029/2012RS005049.

Jacobson, A. R., R. Holzworth, J. Harlin, R. Dowden, and E. Lay, 2006: Performance assessment of the World Wide Lightning Location Network (WWLLN), using the Los Alamos Sferic Array (LASA) as ground truth. J. Atmos. Oceanic Technol., 23, 1082-1092, https://doi.org/10.1175/JTECH1902.1.

Lang, T. J., and S. A. Rutledge, 2008: Kinematic, microphysical, and electrical aspects of an asymmetric bow echo mesoscale convective system observed during STEPS. J. Geophys. Res., 113, D08213, https://doi.org/10.1029/2006JD007709.

,-- , and K. C. Wiens, 2004: Origins of positive cloud-toground lightning flashes in the stratiform region of a mesoscale convective system. Geophys. Res. Lett., 31, L10105, https:// doi.org/10.1029/2004GL019823.

Latham, D., and E. Williams, 2001: Lightning and forest fires. Forest Fires, Academic Press, 375-418.

Lay, E. H., R. H. Holzworth, C. J. Rodger, J. N. Thomas, O. Pinto, and R. L. Dowden, 2004: WWLL global lightning detection system: Regional validation study in Brazil. Geophys. Res. Lett., 31, L03102, https://doi.org/10.1029/2003GL018882.

Lojou, J.-Y., and K. Cummins, 2005: On the representation of twoand three dimensional total lightning information. Conf. on 
Meteorological Applications of Lightning Data, San Diego, CA, Amer. Meteor. Soc., 2.4, https://ams.confex.com/ams/ Annual2005/techprogram/paper_86442.htm.

Lopez, R. E., and R. L. Holle, 1999: The distance between successive lightning flashes. NOAA National Severe Storms Laboratory Tech. Memo. ERL NSSL-1XX, 28 pp., https:// repository.library.noaa.gov/view/noaa/13393/noaa_13393_ DS1.pdf.

Lyons, W. A., M. A. Stanley, J. D. Meyer, T. E. Nelson, S. A. Rutledge, T. L. Lang, and S. A. Cummer, 2009: The meteorological and electrical structure of TLE-producing convective storms. Lightning: Principles, Instruments and Applications, Springer, 387-415.

—, E. C. Bruning, T. A. Warner, D. R. MacGorman, S. Edgington, C. Tillier, and J. Mlynarczyk, 2020: Megaflashes: Just how long can a lightning discharge get? Bull. Amer. Meteor. Soc., 101, E73-E86, https://doi.org/10.1175/BAMS-D-19-0033.1.

Mach, D. M. 2020: Geostationary Lightning Mapper clustering algorithm stability. J. Geophys. Res. Atmos., 125, e2019JD031900, https://doi.org/10.1029/2019JD031900.

—, H. J. Christian, R. J. Blakeslee, D. J. Boccippio, S. J. Goodman, and W. L. Boeck, 2007: Performance assessment of the optical transient detector and lightning imaging sensor. J. Geophys. Res., 112, D09210, https://doi.org/10.1029/2006JD007787.

Marshall, T. C., and W. D. Rust, 1993: Two types of vertical electrical structures in stratiform precipitation regions of mesoscale convective systems. Bull. Amer. Meteor. Soc., 74, 2159-2170, https:// doi.org/10.1175/1520-0477(1993)074<2159:TTOVES >2.0.CO;2.

NOAA, 2018: "When thunder roars, go indoors!"-Lightning safety awareness. NOAA's National Weather Service, accessed 20 June 2020, https://www.weather.gov/ind/LightningSafetyAwareness.

Peterson, M., 2019: Research applications for the Geostationary Lightning Mapper operational lightning flash data product. J. Geophys. Res., 124, 10 205-10 231, https://doi.org/10.1029/ 2019JD031054.

_ 2020: Removing solar artifacts from Geostationary Lightning Mapper data to document lightning extremes. J. Appl. Remote Sens., 14, 032402, https://doi.org/10.1117/1.JRS.14.032402.

_ 2020b: Lightning megaflash data. Harvard Dataverse, V1, accessed 8 September 2020, https://doi.org/10.7910/DVN/ YSDLWJ.

— , and C. Liu, 2011: Global statistics of lightning in anvil and stratiform regions over the tropics and subtropics observed by the Tropical Rainfall Measuring Mission. J. Geophys. Res., 116, D23201, https://doi.org/10.1029/2011JD015908.

, and S. Rudlosky, 2019: The time evolution of optical lightning flashes. J. Geophys. Res. Atmos., 124, 333-349, https://doi.org/ 10.1029/2018JD028741.

,-- , and W. Deierling, 2017: The evolution and structure of extreme optical lightning flashes. J. Geophys. Res. Atmos., 122, 13 370-13 386, https://doi.org/10.1002/2017JD026855.

,-- , and - 2018: Mapping the lateral development of lightning flashes from orbit. J. Geophys. Res. Atmos., 123, 9674-9687, https://doi.org/10.1029/2018JD028583.
— lightning extremes for flash distance $(709 \mathrm{~km})$ and duration (16.73 seconds) recorded from space. Geophys. Res. Lett., 47, e2020GL088888, https://doi.org/10.1029/2020GL088888.

— S. Rudlosky, and D. Zhang, 2020b: Thunderstorm cloudtype classification from space-based lightning imagers. Mon. Wea. Rev., 148, 1891-1898, https://doi.org/10.1175/MWR-D19-0365.1.

Rodger, C. J., S. Werner, J. B. Brundell, E. H. Lay, N. R. Thomson, R. H. Holzworth, and R. L. Dowden, 2006: Detection efficiency of the VLF World-Wide Lightning Location Network (WWLLN): Initial case study. Ann. Geophys., 24, 3197-3214, https://doi.org/10.5194/angeo-24-3197-2006.

Rudlosky, S. D., S. J. Goodman, K. S. Virts, and E. C. Bruning, 2019: Initial geostationary lightning mapper observations. Geophys. Res. Lett., 46, 1097-1104, https://doi.org/10.1029/ 2018GL081052.

Rutledge, S. A., and D. R. MacGorman, 1988: Cloud-to-ground lightning activity in the 10-11 June 1985 mesoscale convective system observed during the Oklahoma-Kansas PRE-STORM project. Mon. Wea. Rev., 116, 1393-1408, https://doi.org/ 10.1175/1520-0493(1988)116<1393:CTGLAI >2.0.CO;2.

Said, R. K., M. B. Cohen, and U. S. Inan, 2013: Highly intense lightning over the oceans: Estimated peak currents from global GLD360 observations. J. Geophys. Res. Atmos., 118, 6905-6915, https://doi.org/10.1002/jgrd.50508.

Schultz, C. J., W. A. Petersen, and L. D. Carey, 2009: Preliminary development and evaluation of lightning jump algorithms for the real-time detection of severe weather. J. Appl. Meteor. Climatol., 48, 2543-2563, https://doi.org/10.1175/ 2009JAMC2237.1.

Stano, G. T., M. R. Smith, and C. J. Schultz, 2019: Development and evaluation of the GLM stoplight product for lightning safety. J. Oper. Meteor., 7, 92-104, https://doi.org/10.15191/ nwajom.2019.0707.

Stolzenburg, M., T. C. Marshall, W. D. Rust, and B. F. Smull, 1994: Horizontal distribution of electrical and meteorological conditions across the stratiform region of a mesoscale convective system. Mon. Wea. Rev., 122, 1777-1797, https://doi.org/ 10.1175/1520-0493(1994)122<1777:HDOEAM >2.0.CO;2.

Williams, E. R., 1998: The positive charge reservoir for spriteproducing lightning. J. Atmos. Sol.-Terr. Phys., 60, 689-692, https://doi.org/10.1016/S1364-6826(98)00030-3.

— - and Coauthors, 1999: The behavior of total lightning activity in severe Florida thunderstorms. Atmos. Res., 51, 245-265, https://doi.org/10.1016/S0169-8095(99)00011-3.

—_, and Coauthors, 2010: Ground-based detection of sprites and their parent lightning flashes over Africa during the 2006 AMMA campaign. Quart. J. Roy. Meteor. Soc., 136, 257-271, https://doi.org/10.1002/qj.489.

Zhu, Y., and Coauthors, 2017: Evaluation of ENTLN performance characteristics based on the ground truth natural and rockettriggered lightning data acquired in Florida. J. Geophys. Res. Atmos., 122, 9858-9866, https://doi.org/10.1002/2017JD027270. 